\title{
Microstructure observation and mechanical behavior modeling for limnetic nacre
}

\author{
Shuchun Zuo • Yueguang Wei
}

Received: 28 February 2007 / Revised: 7 August 2007 / Accepted: 15 October 2007 / Published online: 20 December 2007

(C) Springer-Verlag 2007

\begin{abstract}
In the present research, microstructure of a kind of limnetic shell (Hyriopsis cumingii) is observed and measured by using the scanning electron microscopy, and mechanical behavior experiments of the shell nacre are carried out by using bending and tensile tests. The dependence of mechanical properties of the shell nacre on its microstructure is analyzed by using a modified shear-lag model, and the overall stress-strain relation is obtained. The experimental results reveal that the mechanical properties of shell nacre strongly depend on the water contents of the limnetic shell. Dry nacre shows a brittle behavior, whereas wetting nacre displays a strong ductility. Compared to the tensile test, the bending test overestimates the strength and underestimates the Young's modulus. The modified shear-lag model can characterize the deformation features of nacre effectively.
\end{abstract}

Keywords Nacre $\cdot$ Microstructure $\cdot$ Shear-lag model . Mechanical property

\section{Introduction}

Nacre structure has superior mechanical properties, which is composed of $95 \%$ mineral and 5\% organic (mainly protein and polysaccharide) [1]. Its stiffness is in the same order as that of mineral, and its strength is $2-3$ times higher than

The project supported by the National Natural Science Foundation of China (10432050, 10428207 and 10672163), the Chinese Academy of Sciences (KJCX2-YW-M04) and the Institute of Mechanics through Innovation Project.

S. Zuo · Y. Wei $(\varangle)$

LNM Institute of Mechanics, Chinese Academy of Sciences, 100080 Beijing, China

e-mail: Ywei@LNM.imech.ac.cn that of mineral. Specifically, fracture toughness of the nacre structure is 3000 times higher than that of monolithic mineral $[2,3,5]$. So far the mechanical properties of even man-made ceramic cannot reach such a high level [6,7]. Thus many researches have been carried out on the strengtheningtoughening mechanism of the nacre structure so as to be referred in optimizing design for a new material.

Usually, it is thought that the superior mechanical property of the nacre structure is attributed to its unique microstructure. In last several decades, a lot of researches have been carried out on the microstructure observations, experimental measurement and mechanical behavior modeling for the nacre. Currey first observed the staggered arrangement of nacre platelets and presented a "Brick and Mortar" model to describe the mechanical behavior of the nacre platelets [1]. Wang et al. [8,9] observed the nano-asperities on the platelets and developed a finite element (FE) model based on the friction mechanism. Song et al. [10-12] found the mineral bridges (which are mineral connections crossing protein layer between adjacent mineral platelets) and proposed a "Brick, Bridge and Mortar" model to interpret the strengthening mechanism of nacre structures. Katti et al. [13] developed a platelet interlock model. Barthelat et al. [14] observed a wavy structure and presented an FE model, etc. All above researches are on the sea-shell structures. However, researches on the mechanical property of the limnetic shells are very few. Wang et al. [15] first studied a kind of limnetic shell (Cristaria plicata) using nano-indentation and proposed several strengthening mechanisms based on their observations. They found that there were some distinctions between the sea-shell and limnetic shell structures. The mechanical prosperities were not investigated in their study. Therefore it is significant things to systematically investigate the microstructure feature and the mechanical properties of the limnetic shell. 
In most previous studies on the mechanical properties of nacre structures, the compressive and bending tests were used $[2,4,8]$. Jackson et al. [3] presented a criterion about the spandepth ratio of the beam specimens in the bending tests in order to obtain the effective experimental results. However it was much more complex in plastic case than that in elastic case. There are few researches using tensile test to investigate the mechanical behaviors of the nacre. Currey adopted the tensile test to study the mechanical behaviors of mother of pearl. However only the shape of the load-deformation curve was obtained [1]. Barthelat et al. [14] conducted tensile tests, but the used specimen was so small (the parallel segment is $1.5 \mathrm{~mm}$ ) that the size effect was obvious in their results [14].

In the present study, the microstructure of a kind of limnetic shell (Hyriopsis cumingii) will be observed and measured in detail by using the scanning electron microscopy (SEM) first. Then the mechanical properties of the nacre structure will be measured in both bending and tensile experiments. Moreover, a modified shear-lag model will be used to relate the microstructure of nacre to the overall mechanical properties of the shell, and the modeling results will be compared with the experimental results.

\section{Experimental materials and specimens}

Hyriopsis cumingii - a kind of limnetic shell from the south of China is chosen as the experimental material due to the following considerations: (1) it is a kind of limnetic shell, which is different from the most previous experimental materials (sea-shell); (2) on microstructure features of the shell, the nacre layer is thick and the curvature is small compared with sea-shell; (3) so far there are no reports on the microstructure information and the mechanical properties of Hyriopsis cumingii.

Nacre is a kind of biomaterial which is composed of mineral and protein. The mechanical properties of protein depend on the water contents, so in the present research the specimens are divided into two categories: dry and wetting specimens. Dry specimens are kept in air, while wet specimens are kept in water. Hacksaws and emery clothes are used in specimen machining. The machining rate is controlled and water is used so as to limit the temperature and to guarantee the protein not to be denaturalized under high temperature during the machining. The cross-section of bending specimens is rectangular and the span-depth ratio satisfies the Jackson's criterion. The tensile specimens are processed in the following way. First, a mold of 45 -steel with the same shape and size as the specimen is made by fretsaw. Then the raw nacre material is pasted onto the 45 -steel mold and is ground into the required shape. Fresh fracture surfaces are created for microstructure observations.

Microstructure observations are carried out by using the SEM (FEI Sirion400NC). A Microscale Material Testing
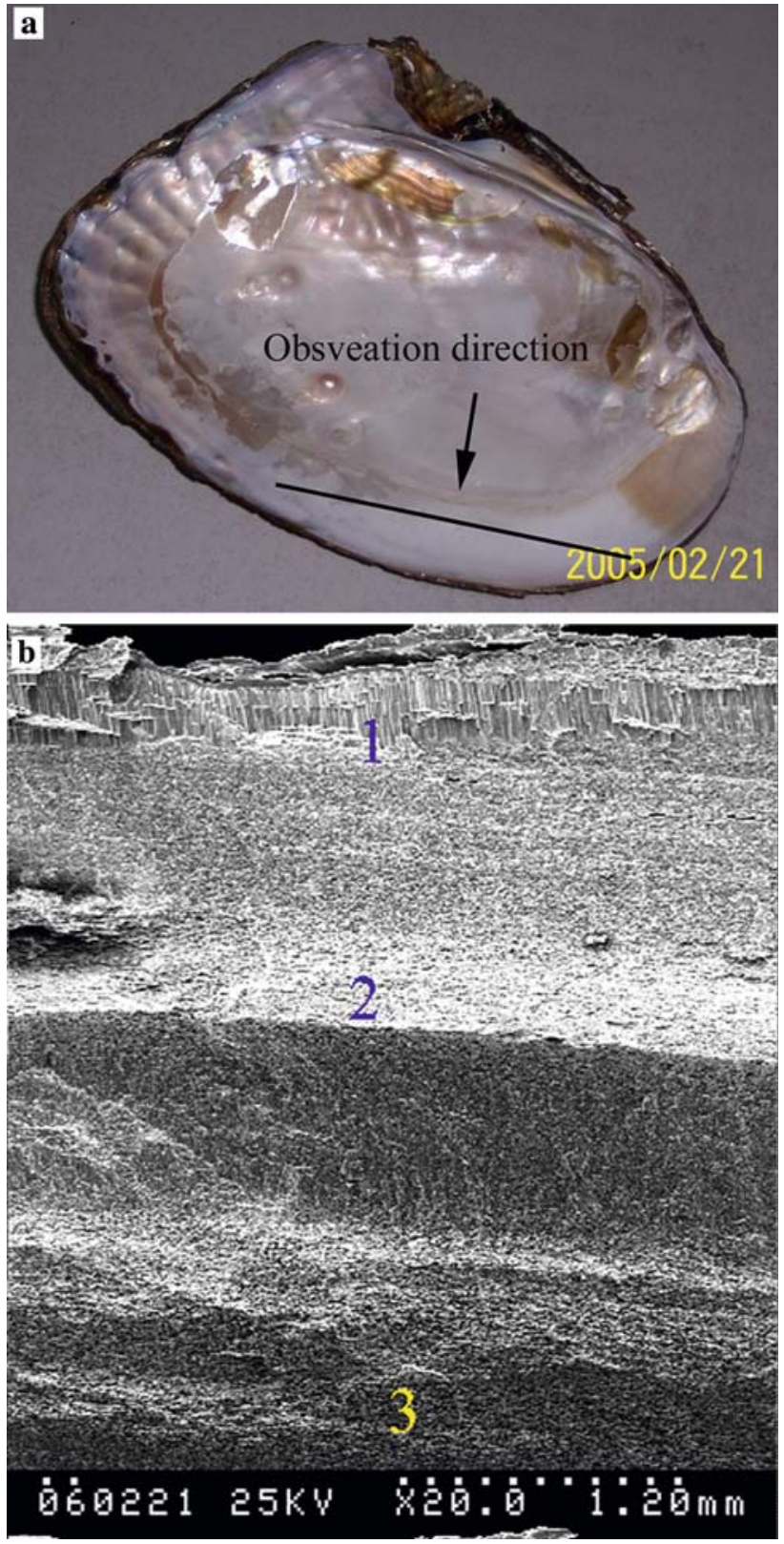

Fig. 1 The shell of Hyriopsis cumingii a and the fracture surface b. Part 1 is the region of prsim layer, where the mineral crystal is like pillars. Parts 2 and 3 are nacres, where the mineral crystal is like platelets

System (MMTS) is used in bending tests. MTS 810 is used in tensile tests. Axial Extensometer $(10 \mathrm{~mm})$ is used for deformation measurements.

\section{Experimental results and discussions}

\subsection{Microstructure feature of shell Hyriopsis cumingii}

The maximum size of the shell is about $190 \mathrm{~mm}$, while thickness is about $3-5 \mathrm{~mm}$ (see Fig. 1a). A fresh fracture surface is made at the position marked by black line, and observation 
Fig. 2 Zooming in the fracture surface: a region $1 ; \mathbf{b}$ region 2; c region 3 in Fig. 1
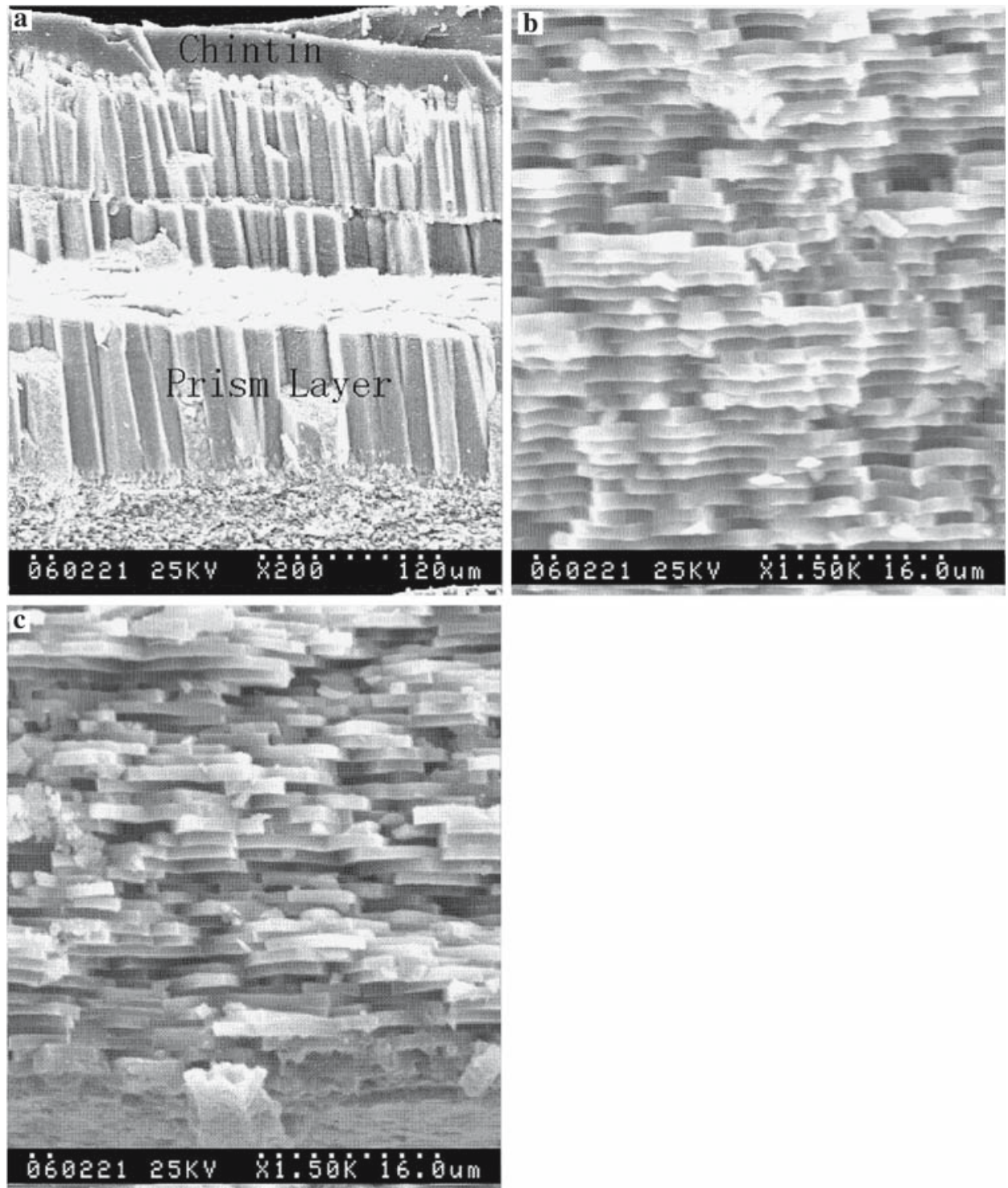

direction is along the arrow, as shown in Fig. 1a. Figure 1b shows the photos of fracture surface with a magnification of 20 . The fracture surface can be distinctly divided into three regions in the thickness direction according to the microstructure features: chitin, prism and nacre regions. It can be seen that the nacre and prism occupy the most area, where the nacre region occupies $80 \%$ of the total area. It is really helpful that the used shell has the high nacre thickness with small curvature.

In Fig. 1b, zooming at 1, 2, 3, we obtain Figs. 2a, b and c, with magnifications 200, 1500 and 1500, corresponding to the chitin, prism and nacre regions, respectively. The chitin layer is located at the outside of the shell, and it is the thinnest layer among all three layers with thickness of about 10 to $20 \mu \mathrm{m}$ (see Fig. 2a). This layer is too thin and weak to have little contribution to the mechanical properties of the shell. The prism layer is located between the chitin and nacre.
It occupies a big region (see Fig. 2b) relative to the chitin layer. This layer is composed of three or four layers of mineral prism crystals with $100 \mu \mathrm{m}$ in height and $15-20 \mu \mathrm{m}$ in diameter. The total height of the prism layer is about several hundred microns. The prism layer has a high compressive strength but a low toughness. The nacre layer is the main structure of shell to bear loads (see Figs. 2b, c). The nacre structure is composed of the staggered platelets in the protein matrix, which forms the "brick and mortar" microstructure. The nacre structure has high stiffness, strength and toughness. The above three-layer structure of shell is typical for most mollusks, but the proportions of the layer thickness are different for different kinds of the mollusks. For the proportion of Hyriopsis cumingii shell, the nacre layer occupies more than $80 \%$ of the total thickness.

It can be seen from Figs. $2 \mathrm{~b}$ and $\mathrm{c}$ that the fracture surface near the outer Fig. $2 b$ is smoother than that near the 
Fig. 3 Microstructure of nacre: a the thickness direction; b The normal direction
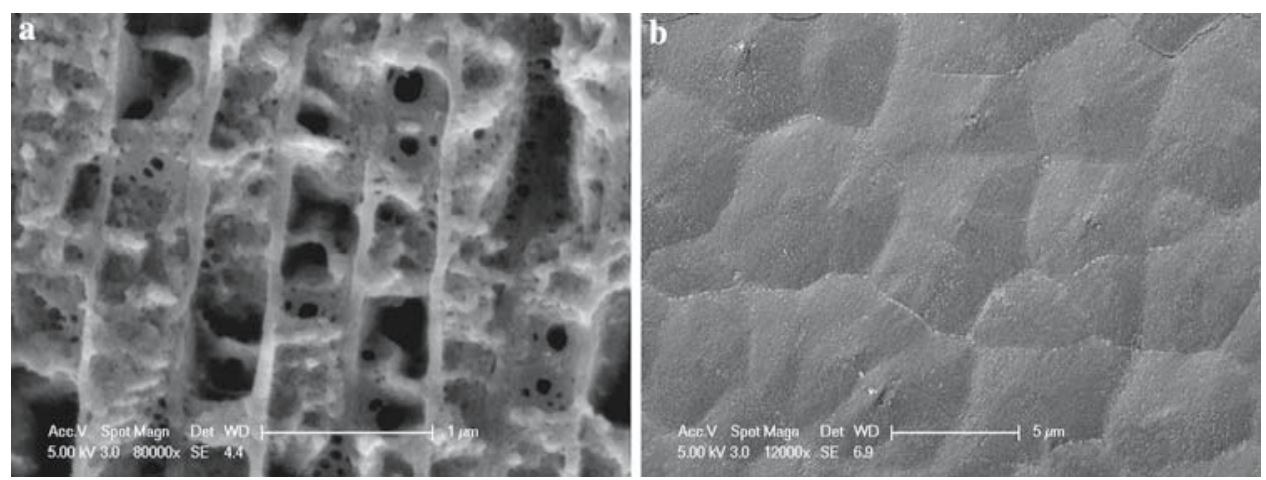

Fig. 4 a Microstructure of nacre platelet, the arrows identify the cracks at the platelet edges; $\mathbf{b}$ the sketch of the analytical model

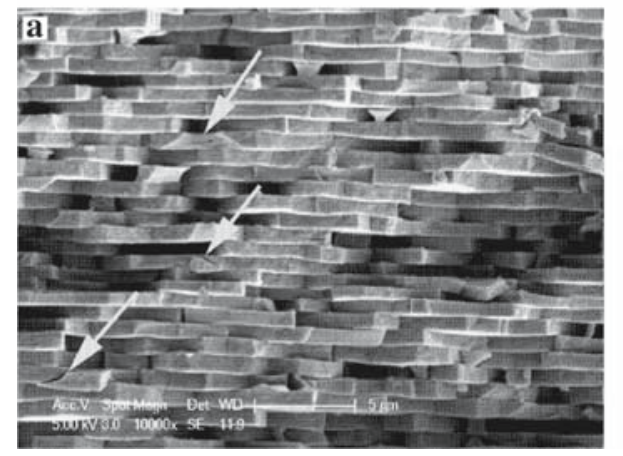

b

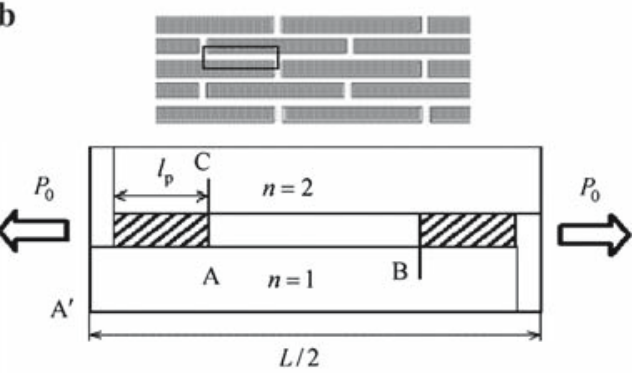

inner Fig. 2c. However, the thickness of individual platelet is almost identical. In order to measure the thickness of the mineral platelets, the fracture surface is polished mechanically and treated with diluted hydrochloric acid. The fracture surface in the same plane of platelets is created and treated with ion beam etching for $5 \mathrm{~min}$ to examine the shape of the platelet.

Figure 3a is the morphology of fracture surface which has been polished and treated with acid. The thickness of platelet in Fig. 3a is about $0.5 \mu \mathrm{m}$ and the thickness of protein between platelets is about $30 \mathrm{~nm}$, which are consistent with that reported in previous articles. The mean diameter of mineral platelets in Fig. $3 \mathrm{~b}$ is about $5 \mu \mathrm{m}$, which is smaller than that of the mollusk shell nacres with a range from 5 to $10 \mu \mathrm{m}$. The shape of platelet is more disorder than that reported in other articles. There are many platelets with triangles or other shapes other than hexagons.

In Fig. 4a, at positions marked with arrows, some cracks on the platelets can be observed. According to previous researches, the platelets are made of $30 \mathrm{~nm}$ column mineral crystals with identical $c$-axis. There is protein at the interface of adjacent mineral crystals [16]. Therefore, the observed cracks must traverse the some column mineral crystals.

\subsection{Mechanical properties}

In the present study, both the bending and tensile experiments will be performed to measure the mechanical properties of the limnetic nacre. For the bending test, Jackson et al. [3] gave a criterion that the span-depth ratio must be larger than 15 in order to obtain the Young's modulus of nacre from the test. Here the size of specimen is 24, 1.6, $4.1 \mathrm{~mm}$ in span, depth and width, respectively, which meets the criterion proposed by Jackson et al. The specimens are cut from the marked position in Fig. 1a. First, the chitin and prism layers are removed mechanically, and then the specimens with the rectangular section are fabricated. Wetting specimens are kept in water for 1-3 weeks. Dry ones are kept in the air. The loading sketch is shown in Fig. 5. From the elastic beam theory, load-deformation curves can be converted into stress-strain curves, as shown in Fig. 5. From the stress-strain curves based on the bending test, the water content remarkably affects the mechanical properties of nacre. For dry nacres the Young's modulus is about $41.4 \mathrm{GPa}$, the strength is about $78 \mathrm{MPa}$, and the maximum strain is only 0.002 , which shows a brittle failure. For wet nacres, when the nacre is kept in water for 1 week, the Young's modulus and the strength are $22 \mathrm{GPa}$ and $69 \mathrm{MPa}$, respectively. The inelastic deformation appears obvious, where the maximum strain is 0.0054 . When the nacre is kept in water for 3 weeks, the Young's modulus and strength are measured as $19.7 \mathrm{GPa}$ and $65 \mathrm{MPa}$, respectively. The maximum strain is 0.0082 . The hardening modulus is $2 \mathrm{GPa}$. It can be seen that the ductility of wetting nacre is much higher than that of dry one, and it increases remarkably with the water content. 


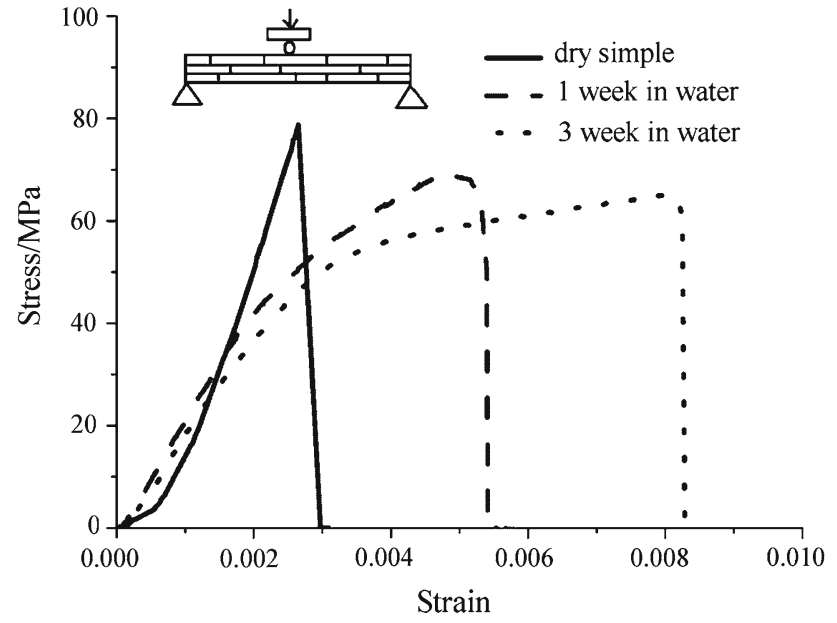

Fig. 5 Stress-strain curves obtained from bending test. The beam span is $24 \mathrm{~mm}$ and rectangular cross-section is $4.1 \mathrm{~mm} \times 1.6 \mathrm{~mm}$

The tensile test is also performed to study the mechanical properties in the present study. The specimens are prepared in the same way as for bending test. Figure 6 shows the tensile specimen before testing. The gauging length of specimen is $15 \mathrm{~mm}$ and the cross-section is $2 \mathrm{~mm} \times 5 \mathrm{~mm}$. The loading rate is $0.1 \mathrm{~mm} / \mathrm{min}$. The stress-strain curve is also shown in Fig. 6 , which is composed of two parts, elastic stage and inelastic stage. One can obtain the material parameters based on the figure, the Young's modulus is $40.9 \mathrm{GPa}$ and the hardening modulus is $8 \mathrm{GPa}$. The maximum strength is about $31 \mathrm{MPa}$. The yield stress is about $25 \mathrm{MPa}$. The maximum strain before fracture is 0.00125 . Compared with bending test results, tensile test results show the higher modulus, lower strength and lower ultimate strain. The strength obtained here is much lower than that given by Barthelat et al. [14] who used the very small specimens for the different kind of shell.

\section{Modeling mechanical properties of the limnetic nacre}

As usual, assume that the deformation mechanism of nacre structure is composed of the mineral tension and protein shear. In the present analysis, we shall adopt a modified shearlag model as shown in Fig. 4b. Referring to the present experimental measurement and experimental result of Menig et al. [4] for protein, assume that the mineral platelet is a linear elastic material, while the protein is an elastic-plastic material with linear hardening. It is worth noting that Gao et al. have proposed a tension-shear model, in which the protein bears linear elastic shear and the shear stress is assumed as uniform distribution in the protein layer. Their result showed that the tension-shear model was better than Mori-Tanaka method for predicting the mechanical properties of nacre [17,18]. Kotha proposed a micromechanical model in which the protein was treated as a linear elastic and perfectly plastic material [19].
In the present model, the linear hardening feature of the protein layer is considered.

Considering that the representative volume element (RVE) is tensioned uniformly on two terminal surfaces, which corresponds to applying a load $P_{0}$ at the terminal surface for the one-dimensional layer model as shown in Fig. 4b, the stress-strain relation will be studied. According to shear-lag analysis, the governing equations due to equilibrium are as follows

$$
\begin{aligned}
& \frac{1}{2} \frac{\mathrm{d} P_{1}(x)}{\mathrm{d} x}+\tau_{1}(x)=0, \\
& \frac{1}{2} \frac{\mathrm{d} P_{2}(x)}{\mathrm{d} x}-\tau_{1}(x)=0,
\end{aligned}
$$

where the origin of coordinate $x$ is located at the left terminal of RVE, and $x$ direction points in the right terminal of RVE. Functions $P_{1}(x)$ and $P_{2}(x)$ are the tensile loads in mineral platelet 1 and platelet 2, respectively, and $\tau_{1}(x)$ is the shear stress in protein. From Fig. $4 \mathrm{~b}, P_{1}(0)=P_{2}(L / 2)=2 P_{0}$.

Because the mineral platelet is linear elastic, we have

$P_{n}(x)=E_{f} h \frac{\mathrm{d} u_{n}(x)}{\mathrm{d} x}$,

where $E_{f}$ is the Young's modulus of mineral, $h$ is thickness of mineral layer. $u_{n}(x)$ is the displacement of mineral platelet $n, n=1,2$ stand for region 1 and region 2 of mineral (Fig. 4b), respectively, $u_{1}(0)=0, u_{2}(L / 2)=u_{0}$, where $u_{0}$ is displacement at the right side terminal surface.

Because the protein is the elastic-plastic material with linear hardening, we have

$\tau= \begin{cases}G \gamma(x) & \tau<\tau_{y}, \\ \tau_{y}+G^{\prime}\left(\gamma(x)-\frac{\tau_{y}}{G}\right) & \tau \geq \tau_{y},\end{cases}$

where $G$ is the shear modulus of protein, $G^{\prime}$ is the linearly hardening shear modulus, $\tau_{y}$ is the shear yield stress of protein, $\gamma(x)=\left(u_{2}(x)-u_{1}(x)\right) / d$ is the shear strain in protein, $d$ is thickness of the protein layer.

Since the maximum shear deformation always occurs at the terminals of the RVE, inelastic (plastic) zones propagate from the terminals of the RVE. The plastic zone size is described by $l_{\mathrm{p}}$, as shown in Fig. $4 \mathrm{~b}$.

By solving above elastic-plastic problem described in Eqs. (2)-(3), one can easily obtain the relationship between the average stress $\bar{\sigma}=P_{0} /(h+d)$ and strain $\bar{\varepsilon}=2 u_{0} / L$.

According to the present experimental observations (see Fig. 4a), in modeling we take $L=3.75 \mu \mathrm{m}, h=0.5 \mu \mathrm{m}$, $d=30 \mathrm{~nm}$. Referring to previous researches $[4,14,20]$, we further take $E_{f}=100 \mathrm{GPa}, G=400 \mathrm{MPa}, G^{\prime}=30 \mathrm{MPa}$, $\tau_{y}=7.5 \mathrm{MPa}$. All parameters here correspond to the wetting nacre. The obtained stress-strain relation from modeling using above parameters is plotted in Fig. 7. For comparison, experimental results are also plotted in Fig. 7. From Fig. 7, it 
Fig. 6 The tensile test specimen and stress-strain relation
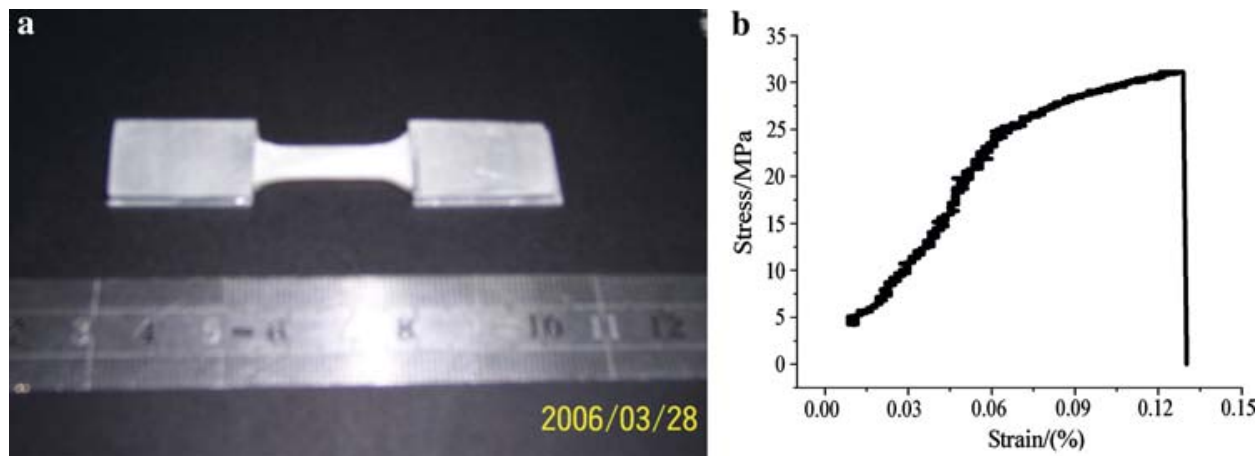

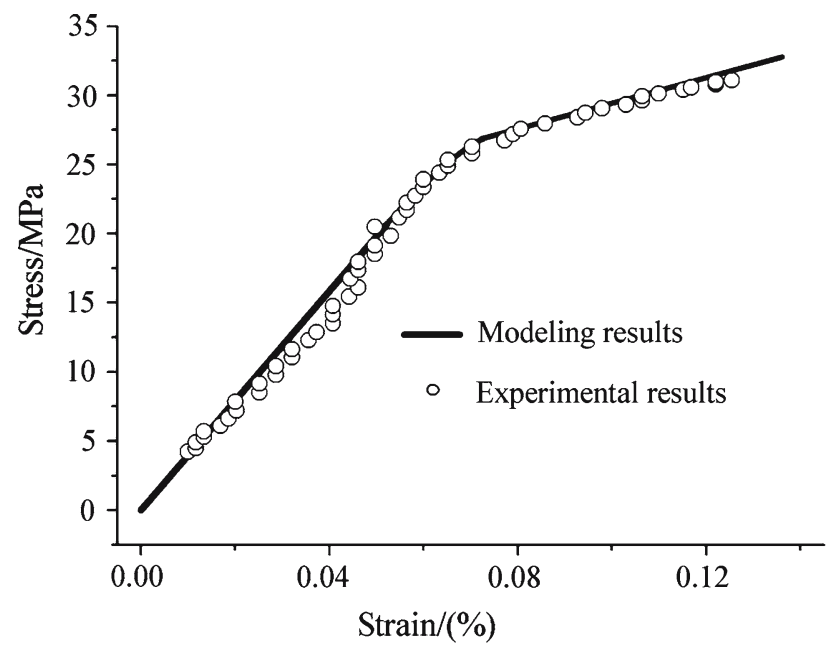

Fig. 7 Stress-strain curves of nacre, comparison of model result with experimental result

can be seen that the model result agrees with the experimental results.

\section{Concluding remarks}

The microstructure observation and mechanical property measurement for a kind of limnetic shell have been performed. The mechanical properties have also been modeled by using a modified shear-lag model. In the modeling, material elastic-plastic hardening behavior has been considered. Through detailed investigation, the following conclusions have been obtained:

(1) Compared with sea-shell structures reported in previous articles, Hyriopsis cumingii as a limnetic shell has a thicker nacre layer, which occupies $80 \%$ of the total thickness, the size of mineral platelets is smaller and the shape is more irregular. However, the overall strength of the nacre is in the same range as other shells.

(2) Mechanical property experiments show strong effects of water contents on the mechanical properties of nacre.
As the water content increases in nacre, the Young's modulus and strength reduce but the material ductility increases. Compared with the results from tensile test, the Young's modulus is underestimated and the strength is overestimated from three-point bending test. There exists an obvious size effect for small specimens.

(3) The modified shear-lag model considering elasticplastic hardening property of protein can describe the nacre deformation well. The modeling results can well fit the experimental results.

It is worth noting that actually the shape and size of mineral platelets are not regular rectangular, and the tensile effects of protein layer, specifically at the end of cell can not be zero, etc, these effects may play an important rule in the mechanical behaviors of the shell, however they are not considered in the modified shear-lag model. Therefore, a further and deep investigation on the mechanical behavior of the limnetic nacre will be necessary in the future.

\section{References}

1. Curry, J.D.: Mechanical properties of mother of pearl in tension. Proc. R. Soc. London B 196, 443-463 (1977)

2. Kamat, S., Su, X., Ballarini, R., Heuer, A.H.: Structural basis for the fracture toughness of the shell of the conch Strombus gigas. Nature 405, 1036-1040 (2000)

3. Jackson, A.P., Vincent, J.F.V., Turner, R.M.: The mechanical design of nacre. Proc. R. Soc. London B 234, 415-440 (1988)

4. Menig, R., Meyers, M.H., Meyers, M.A., Vecchio, K.S.: Quasistatic and dynamic mechanical response of haliotis rufescens (abalone) shells. Acta Mater. 48, 2383-2398 (2000)

5. Menig, R., Meyers, M.H., Meyers, M.A., Vecchio, K.S.: Quasistatic and dynamic mechanical response of strombus gigas (conch) shells. Mater. Sci. Eng. A 297, 203-211 (2001)

6. Almqvist, N., Thomson, N.H., Smith, B.L., Stucky, G.D., Morse, D.E., Hansma, P.K.: Methods for fabricating and characterizing a new generation of biomimetic materials. Mater. Sci. Eng. C 7, 37-43 (1999)

7. Jackson, A.P., Vincent, J.F.V.: Comparason of nacre with other ceramic composites. J. Mater. Sci. 25, 3173-3178 (1999)

8. Wang, R.Z., Suo, Z., Evans, A.G., Yao, N., Aksay, I.A.: Deformation mechanisms in nacre. J. Mater. Res. 16, 2485-2493 (2001) 
9. Evans, A.G., Suo, Z., Wang, R.Z., Aksay, I.A., He, M.Y., Hutchinson, J.W.: Model for the robust mechanical behavior of nacre. J. Mater. Res. 16, 2475-2484 (2001)

10. Song, F., Soh, A.K., Bai, Y.L.: Structural and mechanical properties of the organic matrix layers of nacre. Biomaterials 24, 36213631 (2003)

11. Song, F., Bai, Y.L.: Effects of nanstructures on the fracture strength of the interfaces in nacre. J. Mater. Res. 18(8), 1741-1744 (2003)

12. Song, F., Bai, Y.L.: Mineral bridges of nacre and its effects. Acta Mech. Sin. 17(3), 251-257 (2001) doi:10.1007/BF02486881

13. Katti, K.S., Katti, D.R., Pradhan, S.M., Bhosle, A.: Platelet interlocks are the key to toughness and strength in nacre. J. Mater. Res. 20(5), 1097-1100 (2005)

14. Barthelata, F., Tang, H., Zavattieri, P.D., Li, C.M., Espinos, H.D.: On the mechanics of mother-of-pearl: A key feature in the material hierarchical structure. J. Mech. Phys. Solids 55(2), 306337 (2006)

15. Wang, R.Z., Wen, H.B., Cui, F.Z., et al.: Observation of damage morphologies in nacre during deformation and fracture. J. Mater. Sci. 30, 2299-2304 (1995)
16. Bruet, B.J.F., Qi, H.J., Boyce, M.C., Panas, R., Tai, K., Frick, L., Ortiz, C.: Nanoscale morphology and indentation of individual nacre tablets from the gastropod mollusc trochus niloticus. J. Mater. Res. 20(11), 3157-3157 (2005)

17. Gao, H., Ji, B., JKager, I.L., Arzt, E., Fratzl, P.: Materials become insensitive to flaws at nanoscale:lessons from nature. Proc. Natl. Acad. Sci. USA 100, 5597-5600 (2003)

18. Ji, B., Gao, H.: Mechanical properties of nanostructure of biological materials. J. Mech. Phys. Solids 52, 1963-1990 (2004)

19. Kotha, S.P., Li, Y., Guzelsu, N.: Micromechanical model of nacre tested in tension. J. Mater. Sci. 36, 2001-2007 (2001)

20. Sarikaya, M., Gunnison, K.E., Yasrebi, M., Milius, D.L., Aksay, I.A.: Seashells as a natural model to study laminated composites. In: Biotechnology and Composite Materials, Am. Soc. Comp. Proc. Fifth Tech. Conf., Technomic, Lancaster, PA, 176 pp. (1990) 